\title{
Thyrotoxic Hypokalemic Periodic Paralysis Triggered by Dexamethasone Administration
}

\author{
Waraporn Polamaung ${ }^{1}$, Jaruwan Kongkit ${ }^{2,3}$, Parichat Yimnoi ${ }^{3}$, Patchaya Boonchaya-Anant ${ }^{3,4}$, \\ Thiti Snabboon $3,4, *$
}

\section{ABSTRACT}

Thyrotoxic hypokalemic periodic paralysis (THPP) is a disease characterized by recurrent episodes of muscle weakness due to intracellular potassium shifting in the presence of high levels of thyroid hormone. It occurs more commonly amongst young Asian men with underlying Graves' disease. Attacks are commonly precipitated by ingestion of carbohydrate-rich meals or alcohols, stress or strenuous exercise. Herein, we describe an adult Thai man suffering from a hypokalemic periodic paralysis attack after receiving a dexamethasone injection. The diagnosis of Graves' disease was confirmed by his thyroid function test and a presence of thyrotropin-receptor antibody. His weakness and hypokalemia responded well to potassium supplement and a non-selective beta blocker, while his thyrotoxicosis was initially controlled by an anti-thyroid medication and subsequently with a subtotal thyroidectomy. Clinicians should beware of this manifestation when administering steroids in the thyrotoxic patients, especially of Asian male descent.

\section{KEYWORDS}

thyrotoxic periodic paralysis; hypokalemia; steroid-induced; dexamethasone administration

\section{AUTHOR AFFILIATIONS}

${ }^{1}$ Department of Medicine, Prapokklao Hospital, Chanthaburi Province, Thailand

${ }^{2}$ Department of Medicine, Vachira Phuket Hospital, Phuket Province, Thailand

${ }^{3}$ Department of Medicine, Faculty of Medicine, Chulalongkorn University, Bangkok, Thailand

${ }^{4}$ Excellence Center in Diabetes, Hormone and Metabolism, King Chulalongkorn Memorial Hospital, Thai Red Cross Society, Bangkok, Thailand

* Corresponding author: Excellence Center in Diabetes, Hormone and Metabolism, BhumiSirimangalanusorn Bldg., 4C Fl., King Chulalongkorn Memorial Hospital, Thai Red Cross Society, Rama IV Road, Patumwan, Bangkok 10330, Thailand; e-mail: thiti.s@chula.ac.th

Received: 31 December 2019

Accepted: 26 May 2020

Published online: 10 August 2020

Acta Medica (Hradec Králové) 2020; 63(2): 91-93

https://doi.org/10.14712/18059694.2020.24

(c) 2020 The Authors. This is an open-access article distributed under the terms of the Creative Commons Attribution License (http://creativecommons.org/licenses/by/4.0), which permits unrestricted use, distribution, and reproduction in any medium, provided the original author and source are credited. 


\section{INTRODUCTION}

Thyrotoxic hypokalemic periodic paralysis (THPP) is characterized by recurrent episodes of muscle weakness and hypokalemia from intracellular potassium shifting $(1,2)$. This neurological manifestation is indistinguishable from other subtypes of hypokalemic periodic paralysis except for the presence of thyrotoxicosis state (3). Episodes of attacks can be precipitated by an ingestion of high carbohydrate meal or alcohol, stress or strenuous exercise $(1,3)$. Herein, we describe a patient with THPP attack after receiving a steroid injection and a review of literature.

\section{CASE REPORT}

A 36-year-old Thai man complained of acute weakness in both the lower extremities. Five hours prior, his general practitioner has prescribed him a $5 \mathrm{mg}$ of dexamethasone injection to control his pain from right elbow tendinitis. Thereafter, he developed myalgia which has progressed to paralysis predominantly in both of his legs. He was noted about $4 \mathrm{~kg}$ weight loss and palpitations during the past 6 months. He denied history of alcohol use or a family history of weakness. On examination, the patient was alert with a normal blood pressure and a pulse rate of 115 beats/min. His thyroid gland was slightly enlarged without audible bruit. No exophthalmos was noted. Neurological examination revealed flaccid paralysis and decreased deep tendon reflexes in both the lower extremities. His weakness of $3 / 5$ motor power also involved in proximal part of the upper extremities. Sensory function and cranial nerves were spared. Laboratory results revealed hypokalemia $(2.0 \mathrm{mEq} / \mathrm{L}, 3.5-4.5)$, hypophosphatemia $(2.0 \mathrm{mg} / \mathrm{dL}, 2.5-4.5)$, and mildly elevated serum creatine kinase (300 U/L, 24-195). An electrocardiogram showed sinus tachycardia, a prolonged QTc interval of $0.5 \mathrm{sec}$ and the presence of $U$ wave. His muscle strength and serum potassium were fully restored within 12 hours after the administration of $100 \mathrm{mEq}$ of oral liquid potassium. Rebounded hyperkalemia, $5.8 \mathrm{mEq} / \mathrm{L}$, was noted. Graves' disease was confirmed with $\mathrm{FT}_{4} 1.97 \mathrm{ng} / \mathrm{dL}$ (0.8-1.8), TSH $<0.005 \mu \mathrm{U} / \mathrm{mL}(0.3-4.1)$, and a positive for thyrotropin-receptor antibody. Methimazole $(15 \mathrm{mg} / \mathrm{d})$ and propranolol $(40 \mathrm{mg} / \mathrm{d})$ were administered. One month later, subtotal thyroidectomy was performed uneventfully, and the patient was in euthyroid state and did not experience paralysis during the follow-up period.

\section{DISCUSSION}

Glucocorticoids are currently used in various conditions of Graves' disease including ophthalmopathy due to its anti-inflammatory action; and also in thyroid storm due to its blocking on thyroid hormone peripheral conversion (4). In this report, we demonstrated an unusual complication of glucocorticoid usage in precipitating hypokalemic paralytic attack in a patient with thyrotoxicosis.

Most affected THPP patients are young Asian men (male-to-female ratio of 20-70:1) with underlying Graves' disease despite the female preponderance in hyperthyroidism (male-to-female ratio $1: 4-10)(1-3)$. THPP is also described in any etiology of thyrotoxicosis. The overall incidence of THPP in Asian thyrotoxic patients is about $2 \%$ comparing with $0.1-0.2 \%$ in North America (3). The weakness is characterized by transient and recurrent episodic attacks. It mainly involves the proximal parts of upper and lower extremities and rarely affects bulbar muscles, bowel, bladder or cranial nerves. The severity of weakness usually correlates with the degree of hypokalemia. Sensation is typically intact while deep tendon reflexes are markedly decreased or absent $(1,2)$. The frequency of the attacks can be variable, and the duration of each episode can take from hours to several days. THPP occurs only in the presence of thyrotoxicosis state and sometimes it can be the first manifestation of thyroid disorders as described in our patient. Prodromal symptoms such as cramps or stiffness of the affected muscles are also noted. The attack frequently occurs during the night or in the early morning, and during the resting time. Myalgia has been reported in some cases, in which rhabdomyolysis may occur. Other electrolyte abnormalities that have also been described are hypophosphatemia and hypomagnesemia (5).

The exact mechanism of THPP is still elusive. Hypokalemia from an intracellular shifting is related to an increase in sodium-potassium adenosine triphosphatase $\left(\mathrm{Na}^{+} / \mathrm{K}^{+}\right.$-ATPase) pump activity in the skeletal muscle from a direct stimulation by thyroid hormone, catecholamines, androgens and insulin. This could explain the mechanism of those precipitating factors that can provoke the paralysis attacks from thyrotoxicosis, sympathetic overactivity, hyperandrogenism and hyperinsulinism. In addition, genetic predisposition involving the mutation of transmembrane ion channels of skeletal muscle, including KCNJ18, CACNAIS and SCN4A has been shown in some affected patients (6). Glucocorticoids may induce hypokalemia by directly increasing the $\mathrm{Na}^{+} / \mathrm{K}^{+}$-ATPase pool in skeletal muscle and also by causing hyperinsulinemia. Moreover, steroids can also trigger muscle weakness by inducing myopathy and renal potassium loss owing to its mineralocorticoid effects. From the literature reviews, about 10 cases have been reported regarding the steroids as a precipitating factor of THPP, ranging from low dose prednisolone to pulse methylprednisolone $(7,8)$.

The definitive treatment of THPP is to achieve the euthyroid state. For patients with Graves' disease, an initial treatment with antithyroid drugs followed by radioactive iodine (RAI) therapy or total thyroidectomy is suggested (1-3). According to 2016 ATA guideline, THPP is classified as the clinical situation that favors RAI therapy (4). Nevertheless, our patient chose subtotal thyroidectomy as his preferred treatment. Hypokalemia is usually self-recovery and patients should be monitored for rebound hyperkalemia. Potassium administration during the attacks should be in oral form dividing into small doses with the maximal daily dose of $90 \mathrm{mEq}$ (2). This replacement may shorten the weakness period and prevent the possible fatal arrhythmia; however, it cannot prevent recurrent paralytic attacks. A nonselective $\beta$-blocker, propranolol, is considered to be a first-line agent in the treatment and preventing the paralytic attacks. 


\section{CONCLUSION}

We reported an unusual case of THPP precipitated by the use of a high-dose steroid. Clinicians should beware of the attacks when administering steroids in the thyrotoxic patients, especially of Asian male descent.

\section{ACKNOWLEDGEMENTS}

Some parts of this study have been presented at 41st Annual Meeting of the European Thyroid Association, Newcastle upon Tyne, UK, September 15-18, 2018 as an Abstract-ID: P1-02-14, Steroid-induced Thyrotoxic Periodic Paralysis, A Case report.

\section{REFERENCES}

1. Kung AW. Clinical review: Thyrotoxic periodic paralysis: a diagnostic challenge. J Clin Endocrinol Metab 2006; 91: 2490-5.

2. Vijayakumar A, Ashwath G, Thimmappa D. Thyrotoxic periodic paralysis: clinical challenges. J Thyroid Res 2014; 2014: 649502.

3. Maciel RM, Lindsey SC, Dias da Silva MR. Novel etiopathophysiological aspects of thyrotoxic periodic paralysis. Nat Rev Endocrinol 2011; 7: 657-67.

4. Ross DS, Burch HB, Cooper DS, et al. 2016 American Thyroid Association Guidelines for Diagnosis and Management of Hyperthyroidism and Other Causes of Thyrotoxicosis. Thyroid 2016; 26: 1343-421.

5. Chang CC, Cheng CJ, Sung CC, et al. A 10-year analysis of thyrotoxic periodic paralysis in 135 patients: focus on symptomatology and precipitants. Eur J Endocrinol 2013; 169: 529-36.

6. Rasheed E, Seheult J, Gibney J, Boran G. Does thyrotoxic periodic paralysis have a genetic predisposition? A case report. Ann Clin Biochem 2018; 55: 713-6.

7. Ahamed R, McCalley S, Sule AA. Steroids and Thyrotoxicosis Precipitate Periodic Paralysis. Cureus 2018; 10: e2106.

8. Wongraoprasert S, Buranasupkajorn P, Sridama V, Snabboon T. Thyrotoxic periodic paralysis induced by pulse methylprednisolone. Intern Med 2007; 46: 1431-3. 